\title{
tic\&société
}

Vol. 14, $\mathbf{N}^{\circ}$ 1-2 | 1er semestre 2020 - 2ème semestre 2020

Mutations numériques de la musique : des

contradictions à analyser

\section{Enseigner la guitare sur YouTube. Le tutoriel gratuit et les contradictions de la " numérimorphose » de l'enseignement de l'instrument : le cas de la chaîne MrGalagomusic}

Teaching guitar on Youtube. Free tutorials and contradictions in the "digitalmorphosis" of learning an instrument: the MrGalagomusic channel Enseñal guitarra en YouTube. El tutorial gratuito y las contradicciones de la "digimorfosis" de la enseñanza del instrumento: el caso del canal MrGalagomusic

Raphaël ROTH et Laure-Hélène SWINNEN

\section{OpenEdition}

\section{Édition électronique}

URL : http://journals.openedition.org/ticetsociete/4971

DOI : $10.4000 /$ ticetsociete. 4971

\section{Éditeur}

Association ARTIC

Édition imprimée

Pagination : 187-220

Référence électronique

Raphaël ROTH et Laure-Hélène SWINNEN, « Enseigner la guitare sur YouTube. Le tutoriel gratuit et les contradictions de la « numérimorphose » de l'enseignement de l'instrument : le cas de la chaîne MrGalagomusic », tic\&société [En ligne], Vol. 14, № 1-2। 1er semestre 2020 - 2ème semestre 2020, mis en ligne le 11 novembre 2020, consulté le 23 février 2021. URL : http://journals.openedition.org/ ticetsociete/4971; DOI : https://doi.org/10.4000/ticetsociete.4971 
tic\&société - 14(1-2), 2020

\title{
Enseigner la guitare sur YouTube. Le tutoriel gratuit et les contradictions de la « numérimorphose » de l'enseignement de l'instrument : le cas de la chaîne MrGalagomusic
}

\begin{abstract}
Raphaël ROTH
Raphaël Roth est maître de conférences en sciences de l'information et de la communication au Conservatoire national des arts et métiers (CNAM) - DICEN-IdF (EA 7339), au sein de I'Institut National Supérieur de l'Éducation Artistique et Culturelle (INSEAC) à Guingamp. II s'intéresse à l'étude des publics de la culture, à la musique au cinéma et à l'éducation artistique et culturelle.

raphael.roth@lecnam.net
\end{abstract}

\section{Laure-Hélène SWINNEN}

Laure-Hélène Swinnen est doctorante en sciences de l'information et de la communication sous la direction d'Emmanuel Ethis, Damien Malinas et Raphaël Roth au sein du Laboratoire Culture \& Communication (EA 7542) de l'Université d'Avignon. Son sujet d'étude porte sur l'Éducation Artistique et Culturelle au Festival d'Avignon. laure-helene.swinnen@univavignon.fr 
Enseigner la guitare sur YouTube. Le tutoriel gratuit et les contradictions de la « numérimorphose » de l'enseignement de l'instrument : le cas de la chaîne MrGalagomusic

\section{Enseigner la guitare sur YouTube. Le tutoriel gratuit et les contradictions de la « numérimorphose » de l'enseignement de l'instrument : le cas de la chaîne MrGalagomusic}

Résumé : Cet article présente les résultats d'une étude sur les modalités d'enseignement de la guitare sur YouTube. II met en lumière une des contradictions propres au Do (Learn) It Yourself et à la gratuité. " Je me demande si je n'ai pas tué le métier de prof de guitare en proposant des contenus gratuits ", s'interroge l'auteur de la chaîne MrGalagomusic lors d'un entretien. Comment, alors que la transformation de la consommation de l'écoute musicale intègre une tension qui tend à diminuer, le tutoriel, par sa gratuité, renouvelle-t-il une tension inhérente à la reproductibilité sonore dans le domaine de la transmission des gestes, des savoirs et des connaissances liés à l'apprentissage d'un instrument? Cette tension est propre à la culture de la convergence qu'analyse Henry Jenkins: la contribution d'une communauté par une intelligence collective de l'apprentissage se confronte toujours au problème de la rémunération du créateur. Que devient donc son statut lorsque l'apprentissage est pluriel ?

Mots-clés: YouTube, apprentissage, enseignement, tutoriel, guitare, musique, gratuité. 


\title{
Teaching guitar on Youtube. Free tutorials and contradictions in the "digitalmorphosis" of learning an instrument: the MrGalagomusic channel
}

\begin{abstract}
This article presents the results of a study on how to teach guitar on YouTube. It highlights one of the contradictions inherent in Do (Learn) It Yourself related to free service. "I wonder if I haven't killed the profession of guitar teacher by offering free content?" muses the owner of the MrGalagomusic channel during an interview. Whereas tenisons around listening to music as a consumer activity are decreasing, how do free tutorials renew a tension inherent in sound reproductibility with the transmission of gestures, expertise and knowledge related to learning to play a musical instrument This tension is specific to convergence culture analyzed by Henry Jenkins: the collective contribution of communities to learning always faces the problem of how to pay the creator. What happens then when learning is plural?
\end{abstract}

Keywords: YouTube, learning, teaching, tutorial, guitar, music, free service.

\section{Enseñal guitarra en YouTube. El tutorial gratuito y las contradicciones de la "digimorfosis" de la enseñanza del instrumento: el caso del canal MrGalagomusic}

Resumen: Este artículo presenta los resultados de un estudio sobre las modalidades de enseñanza de la guitarra en YouTube. Destaca una de las contradicciones inherentes al Hágalo Usted Mismo y a la gratuidad. “¿Me pregunto si no he matado la profesión de profesor de guitarra ofreciendo contenido gratuito?" se pregunta el autor del canal MrGalagomusic en una entrevista. ¿Cómo, a la vez que la 
Enseigner la guitare sur YouTube. Le tutoriel gratuit et les contradictions de la « numérimorphose » de l'enseignement de l'instrument : le cas de la chaîne MrGalagomusic

transformación del consumo de la escucha de la música integra una tensión que tiende a disminuir, el tutorial, al ser gratuito, renueva una tensión inherente a la reproducibilidad del sonido en el campo de la transmisión de gestos, conocimientos y habilidades relacionados con el aprendizaje de un instrumento? Esta tensión es específica de la cultura de la convergencia que analiza Henry Jenkins: la contribución de una comunidad a través de una inteligencia colectiva de aprendizaje siempre se enfrenta al problema de la remuneración del creador. ¿Cuál es su nuevo estatus cuando el aprendizaje es plural?

Palabras claves: YouTube, aprendizaje, enseñanza, tutorial, guitarra, música, gratuidad. 
tic\&société - 14(1-2), 2020

\begin{abstract}
La musique est un charme : faite de rien, tenant à rien, peutêtre même qu'elle n'est rien, du moins pour celui qui s'attend à trouver quelque chose ou à palper une chose ; comme une bulle de savon irisée qui tremble et brille quelques secondes au soleil, elle crève dès qu'on la touche; elle n'existe que dans la très douteuse et fugitive exaltation d'une minute opportune. Inconsistante, presque inexistante musique.
\end{abstract}

(Jankélévitch, 2009, p. 149)

\title{
Introduction
}

II semble bien que les descriptions ontologiques sur la musique proposées par le philosophe Vladimir Jankélévitch puissent s'adapter aux conditions dans lesquelles la musique évolue depuis plusieurs années. Avant même de décrire les effets des technologies de l'information et de la communication les plus récentes sur l'enseignement et l'apprentissage de la musique, il nous paraît indispensable de revenir sur quelques spécificités de la musique qui - ineffable avant tout - a toujours, finalement, échappé à toute forme de matérialisation. Cela nous donnera également l'occasion de poser le cadre conceptuel de notre approche et de la situer dans la thématique de ce numéro de la revue tic\&société.

II faut donc commencer par rappeler - sans pour autant nier l'importance des dispositifs numériques et de leurs effets, notamment économiques, sur les objets culturels depuis maintenant plusieurs décennies - que la plupart des modifications majeures de l'écoute musicale que l'on prête parfois au numérique sont bien antérieures à ces dispositifs et datent de l'époque de l'invention de la reproduction sonore à la fin du XIXème siècle (Sterne, 2003). Parmi ces transformations amenées par la seconde révolution industrielle, on notera, en situant les travaux au sein de l'École de Francfort, de Théodor W. Adorno (2010) et de Walter Benjamin (2014): l'ubiquité, l'atomisation de l'écoute, la transformation du statut de l'art en produit de consommation (écoute-marchandise), la perte d'authenticité, la séparation des postures de production et de réception (hic et nunc), etc.

À ce constat s'ajoute le suivant: les technologies d'écoute (appareils de lecture et supports physiques) qui ont précédé le téléchargement de musique et les plateformes numériques 
Enseigner la guitare sur YouTube. Le tutoriel gratuit et les contradictions de la " numérimorphose » de l'enseignement de l'instrument: le cas de la chaîne MrGalagomusic

d'écoute et de visionnage de vidéos en streaming sont toujours utilisées. Malgré l'annonce de sa mort depuis bientôt vingt ans, le disque compact est toujours vivant et le disque vinyle s'offre une nouvelle jeunesse, dans et en dehors des circuits industriels. Selon le Syndicat national de l'édition phonographique (SNEP), 4,1 millions de vinyles se sont vendus en France en 2019, contre 3,9 millions en 2018. Notons qu'en 2015, seulement 900000 exemplaires s'étaient écoulés. Ils séduisent de plus en plus les catégories les plus jeunes, sachant qu'environ $40 \%$ des acheteurs ont moins de trente ans ${ }^{1}$.

De fait, les changements technologiques ont toujours suscité des commentaires relatifs au bouleversement, à la révolution des usages. Cependant, ce dogme de la révolution numérique des usages et des pratiques ne résiste pas longtemps à l'analyse des conditions historiques et sociologiques du développement de ces technologies ni à celle de l'effet de ces technologies sur les pratiques et les usages des consommateurs de culture. En 1981, Jean-Claude Passeron (2006) décrivait déjà les termes révolution et mutation comme des " pierres trop lourdes » qui peuvent venir heurter les pieds des sociologues les employant imprudemment. II expliquait :

Mutation ? Révolution ? II y en a tant eu ici et là, qui n'apparaissent plus, rétrospectivement, dans l'histoire de la culture ou de la technique que comme figures transitoires d'une évolution dont les contemporains se faisaient une idée dont la naïveté et l'émerveillement nous font aujourd'hui sourire. Le désir d'assister en direct à un chambardement qui marquera l'avenir, pour le meilleur ou le pire, est de toutes les époques. Description oui ; prophétie non. (p. 299).

On commence à comprendre le point de vue critique que nous envisageons de développer au sujet du terme numérimorphose, de ses déclinaisons et de ses contradictions : mutations, transformations, métamorphoses, révolution numérique ou, plus proche de notre objet, celui de « musimorphose ».

1 Retrouvez plus d'informations dans: SNEP. (2019). La production musicale française. Décryptage \& performances du marché 2019 de la musique enregistrée. Repéré à https://snepmusique.com/wp-content/uploads/2020/02/bilan-2019-BD2402k.pdf 
Ce que nous décrivons ici pour la musique a investi le champ plus large de l'économie de la culture et ses conséquences sont également connues pour l'apprentissage de la musique, d'autant qu'aujourd'hui, il est possible d'utiliser un même support pour l'écoute de la musique et l'apprentissage de la pratique instrumentale. L'apprentissage de la guitare sur YouTube n'est pas sans poser des problèmes. Tant sur le plan de la pédagogie que sur celui du modèle économique des youtubeurs qui peinent à en vivre sans avoir recours aux " traditionnels » cours à la maison. Nous présentons donc une piste de travail qui n'aura de cesse d'interroger le nouveau et l'ancien, l'innovant et le renouvelé afin de se questionner sur l'état effectif de la " numérimorphose » de l'apprentissage de la guitare avec, en fond conceptuel, la précaution critique quasiment visionnaire rappelée dès 1964 par Adorno : "les mauvais placements de l'industrie culturelle sont aussi extrêmement nombreux et plongent ceux de ses secteurs qui sont dépassés par de nouvelles techniques dans des crises qui sont rarement des chemins vers le mieux » (p. 45).

Partant de l'ensemble de ces constats, il s'agit ici de comprendre comment la forme "tutoriel " sur la plateforme YouTube met en lumière une des contradictions propres au "Do It Yourself » et à celle de la gratuité. " Je me demande si je n'ai pas tué le métier de prof de guitare en proposant des contenus gratuits », s'interroge Éric Legaud lors de l'un de nos entretiens ${ }^{2}$. Comment, alors que la transformation de la consommation de l'écoute musicale intègre une tension qui tend à diminuer (téléchargement légal ou illégal), le tutoriel, par sa gratuité, renouvelle-t-il une tension inhérente à la reproductibilité sonore (Adorno, 2010 ; Miège, 2017) dans le domaine de la transmission des gestes, des savoirs et des connaissances liés à l'apprentissage d'un instrument de musique (Le Guern, 2016) ? Cette tension est propre à la culture de la convergence analysée par Henry Jenkins (2006) : la contribution d'une communauté par une intelligence collective de l'apprentissage se confronte toujours au problème de la rémunération du créateur.

\footnotetext{
2 Entretien réalisé le 30 novembre 2018 au domicile de l'auteur de la chaîne YouTube MrGalagomusic.
} 
Enseigner la guitare sur YouTube. Le tutoriel gratuit et les contradictions de la " numérimorphose » de l'enseignement de l'instrument: le cas de la chaîne MrGalagomusic

\section{La fonction ludique des « tutos » YouTube : définition de l'objet d'étude}

Des recherches récentes en sciences de l'information et de la communication ont établi l'apport des dispositifs ludiques en faveur des apprentissages (Bonfils, Dumas et Massou, 2014). Dans un article récent (Roth et Dufour, 2020), nous nous sommes demandé si nous pouvions réellement bien apprendre sur YouTube par la forme tutoriel, en rappelant que: " La promesse (Jost, 1997) énoncée dans la devise de YouTube "Broadcast yourself" est de permettre aux internautes de "se diffuser eux-mêmes". Cette formulation de YouTube - qui signifie littéralement: "la chaîne vous" - est une promesse partagée ».

Le terme tutoriel qualifie un mode d'emploi d'apprentissage destiné à un utilisateur généralement débutant. II lui permet de se former de manière autonome. Sa forme la plus répandue est la vidéo, mais il peut aussi prendre la forme papier ou celle d'un logiciel. Développé d'abord pour l'apprentissage des logiciels informatiques, le " tuto " s'est élargi à d'autres activités et peut dorénavant concerner tous les domaines de la vie et des loisirs. Ainsi, le tutoriel relève des objets numériques qui sont traversés par les passions ordinaires des amateurs, la « démocratisation des compétences » (Flichy, 2010) et une forme spécifique d'intelligence collective (Jenkins, 2006).

Éric Legaud commente cette connaissance partagée :

« YouTube est une ressource prodigieuse pour n'importe quel apprentissage d'ailleurs. [...] C'est super important d'avoir toutes ces ressources qui sont mises à disposition gratuitement [...]. Les ressources YouTube permettent de s'intéresser à plein de choses maintenant, la philo avec Cyrus North [...]. Chacun avec sa petite connaissance peut apporter sa pierre à l'édifice de la connaissance commune. C'est formidable ! »

\section{MrGalagomusic : privilégier l'approche par cas}

Né à Paris, Éric Legaud commence à étudier le piano dès l'âge de 6 ans puis, en " autodidacte » vers 14 ans, grâce à des magazines spécialisés (il cite Guitar Part), il apprend à jouer de la guitare. À 18 ans, il commence à donner quelques 
cours de musique à domicile, puis dispense son enseignement dans des conservatoires et dans des écoles privées. En 2001, il obtient le $1^{\mathrm{er}}$ prix de piano du Conservatoire international de musique de Paris et, cette même année, entreprend des études en musicologie à l'Université de Paris VIII. Trois ans plus tard, il obtient sa licence. En 2007, il crée une école de musique, Galagomusic, toujours domiciliée à Paris, et c'est en 2011 que la chaîne YouTube MrGalagomusic voit le jour.

La SARL Galagomusic propose des cours gratuits de guitare et de piano sur la chaîne YouTube et rencontre le succès avec, en 2019, 69 millions de vues. En complément, Éric Legaud met à disposition sous la forme de "packs payants " des tutoriels comprenant des vidéos téléchargeables, les partitions correspondantes et un fascicule explicatif. II propose également des cours à distance payants avec le logiciel de visioconférence Skype pour la somme de 30 euros de l'heure. Éric Legaud a en outre publié la première méthode Galago Music aux Éditions Paul Beuscher, intitulée De l'interprétation à la composition (2018). Celle-ci est particulièrement dédiée aux guitaristes ayant au moins un an de pratique. Elle s'accompagne de 2 heures d'audio téléchargeable. II existe aussi les Galago défis, les Galago cover et les Galago reportages que nous n'allons pas développer davantage ici.

Galagomusic est également présente sur Facebook au travers du compte personnel de son auteur, d'une page officielle @MisterGalagoMusic, d'un groupe privé Galagofan créé et administré par des membres de la "communauté » Galago, qui compte plus de 5800 membres sur Twitter et Instagram. Une application disponible pour les systèmes d'exploitation iOS et Android est disponible sur les plateformes dédiées. 
Enseigner la guitare sur YouTube. Le tutoriel gratuit et les contradictions de la " numérimorphose » de l'enseignement de l'instrument: le cas de la chaîne MrGalagomusic

\section{Méthodologie : de l'approche par « cas ॥ au récit de vie $^{3}$}

Ce travail s'appuie principalement sur une analyse de discours de deux entretiens semi-directifs avec le créateur de la chaîne YouTube MrGalagomusic, Éric Legaud. Nous privilégions ici l'approche par cas (Passeron et Revel, 2005) et souhaitons, dans la lignée des travaux menés en sociologie de la réception sur les publics de la culture (Ethis, 2005 ; Malinas, 2008), répondre aux trois principes de méthodes " indispensables pour soumettre à l'observation le projet d'une "esthétique de la réception" " énoncés par le sociologue et épistémologue des sciences sociales Jean-Claude Passeron (dans Passeron et Pedler, 2009, paragr. 1). Nous soulignons :

- celui de perceptibilité d'abord, qui impose à l'enquêteur de n'attribuer à l'objet en question que ses aspects perçus par des publics réels ;

- celui de spécificité ensuite, qui permet d'adapter, dans la méthode d'enquête, l'activité sémiotique d'interprétation et de réception à un terrain et à un cas spécifique ;

- celui de singularité enfin, qui impose de prendre pour objet d'analyse la réception d'œuvres ou de dispositifs particuliers afin de mettre en rapport les comportements et les discours symboliques des publics en rapport avec la structure de l'objet étudié.

Avec ces trois précautions méthodologiques, le recours aux entretiens et à l'analyse des discours de l'enquêté posent le cadre empirique nécessaire à une compréhension de la dynamique de l'enseignement de la guitare sur YouTube, basée sur la méthode du récit de vie, sans prétendre à l'exhaustivité, mais en ne nous empêchant pas quelques exemples empruntés à des cas similaires.

Ce travail complète également un article publié dans la revue Interfaces numériques dans lequel nous réalisons une analyse quantitative de 1867 commentaires d'abonnés ou de visiteurs et d'un corpus de 10 vidéos pour « débutants ou grands débutants " de la chaîne MrGalagomusic d'Éric Legaud (Roth et Dufour, 2020). L'article en question, intitulé « Apprendre la

3 L'article a été relu par Éric Legaud, qui valide les entretiens et reconnaît ses propos. Cette démarche est un point de méthode essentiel de notre approche. 
guitare sur YouTube ", a permis d'établir une taxonomie de commentaires (Paveau, 2017) (félicitations, remerciements, qualité pédagogique, commentaires sur le youtubeur, demande d'un morceau, parmi les catégories les plus présentes) et de comprendre comment la forme tutoriel permet le partage d'expériences, de savoirs, de compétences qui ne relèvent pas du schéma "classique " de la transmission en sciences de l'information et de la communication.

Le "tuto " sur YouTube engage une communication du youtubeur vers l'internaute, de l'internaute vers le youtubeur ou de l'internaute vers un autre internaute par la possibilité de laisser un commentaire et d'y répondre. II s'apparente davantage aux formes de communication orchestrale qui reposent sur l'interaction et constitue un objet pertinent pour comprendre les logiques de partage, d'échange, de contribution et de participation engagées sur les interfaces numériques (Roth et Dufour, 2020). Une des pistes évoquées, mais non développée dans cette recherche, concerne une tension inhérente sur Internet à la numérisation des contenus culturels, à savoir celle de la gratuité. Nous nous contenterons d'aborder cette question de la gratuité dans le cas qui nous intéresse. En effet, nous souhaitons, dans le présent article, comprendre comment, du point de vue de l'enseignant, se fixent des contradictions entre gratuité et valorisation marchande des ressources éducatives sur YouTube.

\section{Gratitude de la gratuité : première hypothèse}

L'analyse des commentaires atteste que si la reconnaissance passe par la gratuité de la contribution, cette gratuité n'est pas énoncée comme un avantage par les internautes eux-mêmes. En effet, sur les 1867 commentaires issus du corpus analysé, seulement quatre font mention de l'adjectif gratuit. Un seul utilise l'adjectif payant.

Nous les restituons ici dans leur intégralité :

Vidéo Personal Jesus (Johnny Cash) - Tuto quitare bluesy

Commentaire $\mathrm{n}^{\circ} 1$ - Quentin Dupouey - 16-10-2018 14:13 : « c'est top ce que tu fais ! je m'en vais de ce pas sur PayPal :) » 
Enseigner la guitare sur YouTube. Le tutoriel gratuit et les contradictions de la "numérimorphose » de l'enseignement de l'instrument : le cas de la chaîne MrGalagomusic

Commentaire $\mathrm{n}^{\circ} 53$ - Emberi - 16-04-2016 - 10:05: «POURAUOI TON PACK PAYANT EST SUR LE REGGAE TAURAIS DU FAIRE REGGAE GRATUIT ET ROCK PAYANT !!! »

Commentaire $n^{\circ} 54$ - MrGalagoMusic - 17-04-2016 19:31 : "+thesame56 J'ai fait aussi du reggae gratuit. Et enlève le Caps Lock. »

Vidéo Comment accorder sa gratte - Cours de quitare

Commentaire $n^{\circ} 37$ - Z3Rod ! Vidéaste - 13-06-2016 21:11: " je suis totalement d'acc, perso j'utilise GuitarTuna depuis un bon moment déjà et cette appli est vraiment top, accordeur que l'on peut augmenter en sensibilité sonore, métronome, dictionnaire de tous les accords en plus l'appli est gratuit ! »

Commentaire $n^{\circ} 175$ - KGBIngenius - 25-05-2012 12:43 : « Vraiment cool de faire ça gratuitement continue $\wedge \wedge$ ”

Vidéo Hallelujah (Leonard Cohen) - Tuto guitare (UK Subtitles)

Commentaire $n^{\circ} 5$ - Paul Savagner - 06-07-2018 17:10 : «Bonjour, merci énormément pour tes vidéos. Merci beaucoup de ne pas pousser des liens vers des sites payants dans tes vidéos, ça rend l'expérience d'apprendre la guitare avec toi beaucoup plus sympa que sur d'autres chaînes (en plus du fait que tu es un prof génial ). Merci pour tout! »

Commentaire $n^{\circ} 51$ - Tzunami06fr - 10-12-2016 - 10:59: « C est tres con faire payer la tablature (4 euros!) surtout qu'on la même gratuitement un peu partout... »

Vidéo: Calvin Russel (Rats and Roaches) - Débutant Blues 12 mesures en Mi - Facile et Indispensable!

Commentaire $n^{\circ} 64$ - Oxydes - 07-09-2018 - 21:43: "Incroyable qu'une personne mette autant de tutos a disposition gratuitement! Tu pourrais ouvrir un UTip afin d'être rémunéré avec des dons ou en visionnant des publicités ? C'est dommage que tu ne sois pas vraiment rémunéré pour autant de travail.. » 
Commentaire $n^{\circ} 66$ - Yannick B - 09-09-2018 - 19:47: « II y a le GALAGOTHON si tu veux le remercier, va voir sur son site galagomusic Je le suis depuis un petit moment, et c'est de loin le plus actif en gratuit avec Guitorama sur YouTube aussi. Pas d'actions chez l'un ou l'autre, mais ils sont top et gratuit tous les 2 ;-) »

Du point de vue des amateurs de tutoriels, la gratuité est rarement un sujet de commentaires et de discussions. Elle relève de la gratitude exprimée par les remerciements (39\% des commentaires du corpus comprennent le mot merci) et les félicitations $(31 \%$ des commentaires du corpus). Les félicitations sont associées aux remerciements. Le youtubeur apporte un bienfait dont la force symbolique réside notamment dans la gratuité de sa contribution. Le partage d'une connaissance, d'une compétence, d'un geste, d'un "truc » s'appuie sur la reconnaissance d'une compétence, d'une expertise qui légitimise le statut du "prof » et son succès. II semble que la gratuité relève d'une évidence: de la normalité de l'accès aux contenus sur Internet. Accéder à un cours de guitare gratuitement serait-il donc chose normale? Tout concourt à asseoir cette normalité de la consommation gratuite des contenus sur Internet: la facilité d'accès à ces substituts éducatifs, instructifs et didactiques n'importe quand, n'importe où et quel que soit le support: téléphone multifonctions, ordinateur, tablette accentuent la «consommation personnalisée " que nous avons pu décrire par ailleurs (Leveratto, Pourquier-Jacquin et Roth, 2015).

\section{Première contradiction : « si c'est gratuit, c'est que c'est vous le produit "}

La gratuité peut cependant être une promesse énoncée comme un produit d'appel. Parmi les nombreux groupes et publicités Facebook qui existent sur l'apprentissage de la guitare, nous découvrons une publicité de « Jejouedelaguitare.com » qui compte 93042 vues, 183 partages et 684 réactions. En portant un regard sur ces commentaires relationnels (le «like» Facebook), conversationnels ou partages (Paveau, 2017), une interaction attire notre regard (Figure 1). 
Enseigner la guitare sur YouTube. Le tutoriel gratuit et les contradictions de la " numérimorphose » de l'enseignement de l'instrument: le cas de la chaîne MrGalagomusic

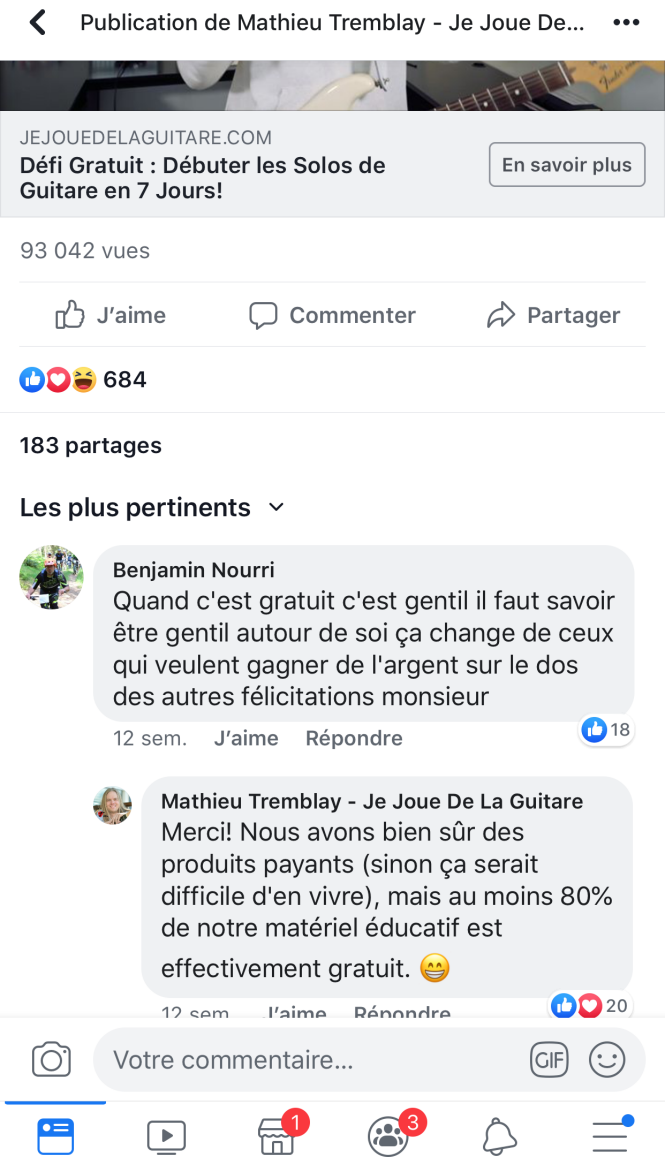

Figure 1. Capture d'écran de commentaires Facebook Jejouedelaguitare.com

L'échange présenté dans la figure 1 entre un abonné de la page Facebook de la chaîne "Je joue de la guitare " et Mathieu Tremblay, le professeur de guitare de la chaîne, est emblématique de ces tensions. Elles ne concernent pas uniquement le cas de la musique et de sa consommation : souhaiter, sans y parvenir, consulter un article de presse dont le contenu principal est réservé aux abonnés est chose courante sur les réseaux socionumériques et les commentaires associés à l'expression du mécontentement sont faciles à trouver.

C'est une stratégie qui repose sur la frustration : un contenu qui semble gratuit, mais qui ne l'est pas. Ici, la tension « gratuit/payant » est présente et énoncée par les internautes. 
Le tutoriel, outre sa fonction d'enseignement et sa dimension ludique, possède donc une vertu promotionnelle. La promotion et la publicité sont le pendant de la gratuité. "Si c'est gratuit, c'est que c'est vous le produit »: la formule se retrouve dans différents articles de presse et blogues sur Internet.

Dans le cas d'Éric Legaud, dont l'enseignement de la musique est le métier, la chaîne YouTube relève d'une prolongation de sa professionnalisation dont le tournant s'est réalisé lorsqu'il a créé son entreprise :

« J'ai commencé à donner des cours à partir de mes 18 ans. [...] Et en 2007, je me suis dit que j'en avais marre d'être payé 5 euros l'heure [...]. Et j'ai ouvert ma boîte : une école de musique qui s'appelait GalagoMusic [...]. C'est en 2011 que j'ai ouvert la chaîne YouTube "MrGalagoMusic". C'est une continuité de mon métier [...], j'ai juste fait ce que je sais faire en fait. »

YouTube est d'abord envisagé comme une continuité, comme la prolongation sur un support différent, du métier initial. II peut tout autant être considéré comme un outil promotionnel qui repose sur la reconnaissance et qui renforce la légitimation. Il fonctionne comme " une carte de visite ".

«Quand je poste une vidéo, là j'arrive à 280000 abonnés il me semble, ça paraît bien. En fait, ce qui est cool, c'est que ça installe une crédibilité ; vis-à-vis des gens. Oh j'ai 280000 abonnés ça fait une carte de visite intéressante. »

\section{Seconde contradiction : le succès de la chaîne YouTube ne rime pas forcément avec le succès des cours à domicile}

Cependant, YouTube n'est pas l'eldorado imaginé, notamment en ce qui concerne l'attrait pour les cours individuels "classiques » au domicile de l'enseignant ou de l'élève.

« Je suis toujours un peu arrogant - mais je me dis [...] je vais trouver du monde. En un an et demi, je galère à faire encore 10 élèves par semaine. À Paris, j'en avais 50, et j'aurais pu en avoir $80[\ldots]$. C'était très dense. Mais bon, c'est vrai que là c'est un grand écart... » 
Enseigner la guitare sur YouTube. Le tutoriel gratuit et les contradictions de la "numérimorphose » de l'enseignement de l'instrument : le cas de la chaîne MrGalagomusic

En effet, un grand nombre d'abonnés n'offre ni automatiquement ni systématiquement la garantie pour Éric Legaud de remplir son carnet de rendez-vous concernant les cours à domicile :

"Quand on est arrivé ici [...], j'ai fait une petite vidéo devant le pont d'Avignon [...] qui disait "[...] Retrouvez-moi sur le site ou Facebook, je vais reprendre mes activités comme je faisais à Paris. Je donne des cours. Si vous êtes intéressés, envoyez un mail". J'ai eu 4-5 réponses. Ce n'était pas beaucoup. Et sur les 4-5, je n'ai eu que 2 cours [...]. Puis après, tous les autres contacts se sont faits via Facebook parce que du coup j'ai relancé [...]. Quand tu as deux-trois personnes sympas qui sont contentes, elles en parlent avec deux autres personnes qui vont en parler avec d'autres. Donc après, c'est le réseau qui fait le truc. Mais même avec ce réseau, c'est très restreint. À dix, c'est vraiment une semaine géniale. Je me suis dit [...] : "Je vais penser le dispositif autrement. Qu'est-ce que j'ai à disposition: des réseaux sociaux et un savoir-faire. Qu'est-ce que je peux faire ?" Les vidéos YouTube, ce n'est pas que ça rapporte. C'est vrai que ça commence maintenant à payer un petit peu [...], je me suis dit: "pourquoi pas faire des cours par Skype ?" [...] J'ai mis en ligne un planning électronique qui dit [...] les plages horaires qui sont disponibles. Une fois que t'as réglé, ton créneau est réservé, on se retrouve à telle heure pour faire un cours via la caméra [...]. Donc là pareil, toujours avec mon arrogance je me suis dit: "ça va être plein tout de suite". En deux semaines j'avais 0 réservation [...] j'avais, il y a un an, 250000 abonnés. On peut se dire que ça fait du monde. [...] II y a quand même un sujet "qu'est-ce que ça prouve vraiment le terme d'abonnés sur YouTube ?" "

Le premier élément à souligner pour analyser ce qui vient d'être décrit est la discordance qui existe entre le nombre d'abonnés, le nombre de vues et la notoriété des vidéos. Plus de 300000 abonnés de la chaîne ne rapportent que rarement plus de 10000 ou 20000 vues en un week-end, même en proposant un contenu varié :

"Après, il y a peut-être un désintérêt des tutos [...]. Parce que je vais voir la chaîne des collègues [...], il y en a un 
qui s'appelle Justin Sandercoe qui a une grosse chaîne. II a presque 900000 abonnés ou presque un million, je crois. Quand il poste une vidéo, il va faire 25000 vues. Ce qui n'est pas bien. Quand tu as un million d'abonnés et que tu as 25000 vues, tu n'es pas content. »

Le succès médiatique du youtubeur favorise sa reconnaissance, mais c'est la diversification des supports (cours à domicile, cours à distance synchrones par Skype ou asynchrones par packs payants, méthode de guitare au format papier) de cours et les dons qui permettent à l'enseignant de vivre de son métier de professeur de guitare.

« RR : Tu as une offre payante de cours?

EL : [...] J'ai 10 packs payants.

$\mathrm{RR}:$ Ça te rapporte?

EL : Les packs payants, oui. C'est vraiment le truc qu'il ne faut pas que je rate [...]. J'en sors 2 par an : un au mois de juin et un au mois de décembre. Et ça me fait à peu près un quart de mon bilan [...].

RR : YouTube gratuit, ta chaîne, ça t'amène...

EL : Ça amène des gens justement à acheter ces trucs-là. Quand ils sont contents du gratuit [...]. En fait, je fais un business [...] qui est construit sur la reconnaissance. Je me suis toujours dit si les gens ils voient que je me casse la tête à faire des trucs de qualité [...] au bout d'un moment ils vont se dire on va l'aider en retour. C'est un peu ce qui se passe. Mais c'est vrai que par rapport à la masse de gens qui a dans les abonnés [...] le résultat peut être jugé un peu décevant. [...] je travaille à faire en sorte qu'au bout d'un moment ça puisse vraiment devenir rentable $[\ldots]^{4}$. »

\section{Troisième contradiction : la monétisation YouTube ne suffit pas à rémunérer l'auteur et les politiques en la matière varient}

L'impératif de rentabilité qui doit permettre à l'auteur de vivre de son métier résonne comme une contradiction flagrante si

${ }^{4}$ Extrait d'un entretien entre Éric Legaud (EL) et Raphaël Roth (RR). 
Enseigner la guitare sur YouTube. Le tutoriel gratuit et les contradictions de la "numérimorphose » de l'enseignement de l'instrument : le cas de la chaîne MrGalagomusic

l'on considère qu'Éric Legaud propose $98 \%$ de contenu gratuit et seulement $2 \%$ de payant :

« J'ai, vraiment à reculons, commencé à parler de contenu payant. Je n'osais pas en fait [...]. Donc je me débrouillais autrement pour faire passer mes charges, mais [...] j'étais gêné. Et puis plus tu avances, tu te dis [...] c'est normal, tu n'obliges pas les gens à acheter. Les tutos gratuits restent gratuits. »

Comme d'autres vidéastes du web, Éric Legaud rencontre des difficultés sur YouTube avec les questions de droits à la propriété intellectuelle ou droits d'auteur :

«Mes vidéos sont régulièrement démonétisées. Les droits vont déjà aux auteurs. L'avenir de ma chaîne YouTube et de la chaîne de plein d'autres youtubeurs [...] est en suspens."

"La Sacem [Société des auteurs, compositeurs et éditeurs de musique] te dit "tu expliques le morceau de quelqu'un, tu utilises la grille d'accord d'untel, la parole d'untel, ça ne t'appartient pas que je sache". Tu dis effectivement non, la tête baissée [...], je ne toucherai rien là-dessus. "

Malgré ses différentes sources de revenus, la variété de son offre, son dynamisme et sa volonté d'entreprendre, Éric Legaud admet qu'il souffre de ce manque de visibilité et de reconnaissance. II reconnaît ses difficultés à réussir dans un domaine d'activité encore balbutiant, mais prometteur et concède que l'économie numérique tarde à tenir toutes les promesses qu'elle a générées.

« J'ai plusieurs sources de revenus qui me permettent de faire fonctionner ma boîte, et j'espérais à un moment donné que YouTube puisse me rapporter au moins 1000 euros par mois. [...] Sur l'année, ça aurait complété les cours Skype ; avec les dons aussi. Parce que les dons sont quand même des sources de revenus qui s'élèvent entre 200 et 300 euros par mois [...]. Et là j'étais en chute libre dans mes scores. Quand t'es youtubeur, tu regardes beaucoup tes scores. [...] C'est un indicateur. C'est mon thermomètre qui me permet de me dire "bon, les gens sont contents, je fais du bon travail". Je ne sais pas ce qui 
s'était passé entre mars et août, j'ai perdu à peu près $60 \%$ de mes vues. II y a eu une chute impressionnante. Janvier 2018, j'étais arrivé à un score de ouf : 1300000 vues par mois. J'étais content. »

« En tout cas, ça s'est maintenu pendant un mois ou deux: le fait d'avoir dépassé 1 million d'abonnés [...] j'avais réussi [...]. Et tout s'est brisé. J'ai vu les courbes descendre comme si j'étais à la bourse... Ça ne représente pas mon argent, mais ça représente l'investissement [...]. ॥

Ce faisant, au regard des résultats aléatoires de sa chaîne, l'enseignant revient toujours à son métier initial :

« RR : [...] tu as sorti une méthode, Paul Beuscher ${ }^{5}$ c'est ça?

EL : Exactement.

$\mathrm{RR}$ : Tu peux m'en parler un peu [...] ?

EL : C'est Beuscher qui m'a contacté il y a 1 an et demi. II m'a envoyé un message en me disant "on sait ce que vous faites, ça nous intéresserait de bosser avec vous" [...] Paul Beuscher, j'ai acheté ma première guitare chez eux, j'ai acheté toutes mes patoches de conservatoire chez eux, c'est rigolo quand même. Donc, allons-y. Et j'ai essayé de penser une méthode [...] c'est tout plein de conseils [...], des petites techniques clés en main. Ça peut à la fois résumer ce qu'on trouve en gratos avec du contenu totalement original [...].

$\mathrm{RR}$ : Ça te rapporte un peu d'argent?

EL : 1,60 euro par exemplaire vendu. Donc $6 \%$. Et on en a vendu à peu près 2500. [...] lls sont très contents parce qu'apparemment sur un produit comme ça ils en vendent 300 [...] ça me fait plaisir. Ça fera des sous qui rentrent, mais même si effectivement sur un produit qui coûte 23 euros je n'en rentre que 1 euro [...]. Ils m'en ont commandé une autre pour le piano. Je pense qu'il y a moyen de faire quelque chose de vraiment original qui soit complémentaire de ma vidéo [...]. La différence avec mon

${ }^{5}$ Entreprise d'instruments de musique et d'édition musicale. 
Enseigner la guitare sur YouTube. Le tutoriel gratuit et les contradictions de la " numérimorphose » de l'enseignement de l'instrument: le cas de la chaîne MrGalagomusic

truc à moi, c'est qu'effectivement je le vends 20 euros et j'ai 20 euros dans la poche. Enfin, tu enlèves la TVA...

$\mathrm{RR}$ : Tu en vends 2500 de ton pack?

EL : J'aimerais bien. Mais non, tu imagines 2500 à 20 euros, ce serait la fête ! [...] J'en vends 200 . Si je suis très très bon, j'en vends 300 . En gros, pour la fin d'année il faut que j'arrive à rentrer 500 pour faire mon chiffre. Donc, je vais me débrouiller. Je vais faire de la pub sur Facebook et d'autres plateformes. La semaine dernière j'étais invité chez RTL2 parce qu'ils avaient besoin d'un prof pour un concours lancé par Charlie Winston. J'utilise maintenant toutes ces particularités, toutes ces rencontres [...]. Je vais essayer vraiment de les mettre en avant pour crédibiliser mon travail YouTube pour offrir une image qui soit absolument professionnelle. J'aimerais bien devenir un référent. Tu as besoin d'un prof de guitare, c'est Galago. [...] II faut encore que je travaille. ॥

Si le passage au numérique de l'enseignement de la guitare a autorisé et favorisé la gratuité, il a également contribué au succès du tutoriel. Cependant, la réalité de la professionnalisation de l'enseignement est toute autre. Les stratégies mises en place par le professeur de guitare qui choisit YouTube ne relèvent pas uniquement de l'altruisme et du plaisir gratuit de transmettre. Le paradoxe tient, nous l'avons dit, dans la promesse à la fois du tuto et d'Internet : la gratuité. Pour conserver cette promesse et tenir sa communauté, le professeur sur YouTube doit se diversifier. Cette situation est partagée par d'autres youtubeurs professeurs de guitare :

« RR : Parmi les youtubeurs en France ou à l'étranger il y en a qui t'inspirent?

EL : Celui qui m'a inspiré, qui m'a donné envie d'ouvrir ma chaîne YouTube c'est Marty Schwartz. [...] C'est le premier américain [...] ça fait peut-être 12 ans qu'il fait ça bien avant tout le monde [...]. Déjà il installe un personnage sympathique [...]. Surtout il propose des vidéos sur les solos que personne n'avait expliqués avant. Avec vraiment des consignes simples. [...] II m'a appris des tonnes de choses. [...] Par contre, j'avais trouvé qu'il n'expliquait pas très bien les morceaux. Là, je me suis dit que moi je sais le faire, je pense que je peux le faire et 
peut-être mieux que lui. Et du coup, j'ai commencé ma chaîne comme ça. [...] Par contre, Justin Sandercoe qui est anglais, qui fait du très bon travail. Lui, c'est pareil. II est très connu [...]. Et maintenant l'étoile montante c'est Paul Davids [...]. II propose du contenu qu'on ne trouve pas ailleurs $[\ldots]$.

RR : Ça ne suffit pas pour être un bon prof de guitare en fait ? II faudrait plus que ça ?

EL : Ben ouais. C'est-à-dire si tu es juste bon, ce n'est pas suffisant sur YouTube. Maintenant on est trop. En 2007, oui, si tu étais juste bon, OK. Maintenant il y en a plein qui sont très bons, mais qui n'arrivent pas pour autant à sortir parce que soit ils n'arrivent pas à trouver leur personnage à eux, soit ils n'arrivent pas à trouver une méthode originale d'expliquer. Tout est à construire. Tout est à imaginer sur le tuto. [...] C'est protéiforme en fait. [...] L'autre fois j'ai fait un tuto sur Bob Marley [...] c'était sur Jammin. J'ai fait un tuto basse, guitare et piano, exprès pour jouer un groupe. Ça n'existe pas. Tous ces trucs-là qui ont beau être originaux et je trouve qu'ils étaient de très bonne facture, n'ont pas eu - celui de Bob Marley notamment - l'écho que j'espérais [...]. »

\section{Quatrième contradiction : les dispositifs numériques créent un sentiment de communauté qui a besoin de se rassembler physiquement}

Une des tactiques envisagées par Éric Legaud est de passer outre les contraintes matérielles que lui imposent les écrans et, estimant que rien ne peut véritablement supplanter le contact humain et la communion interactive du groupe, de se rapprocher physiquement de sa communauté. Ainsi, après avoir voulu mettre fin à sa chaîne, il propose :

« J'ai tout remis en question [...], j'ai posté une vidéo en septembre, en disant voilà je pense que c'est la dernière année où je fais quelque chose sur YouTube [...], mais je vais quand même laisser un an de plus, on va bien voir. En revanche cette année, je vais tout faire pour que ça déchire [...]: contacter des médias, contacter des journaux, des magazines, des artistes tout ça. Et j'ai voulu 
Enseigner la guitare sur YouTube. Le tutoriel gratuit et les contradictions de la "numérimorphose » de l'enseignement de l'instrument : le cas de la chaîne MrGalagomusic

aussi pour casser cette espèce d'hermétisme qu'il y a entre le fait d'enregistrer ou d'avoir une caméra et d'être tout seul tout le temps. Parce que je m'adresse à plein de gens, mais je ne les vois pas [...]. lls me laissent des messages. C'est bien, mais c'est dématérialisé. II n'y a aucun contact. Donc je me suis dit cette année, je vais proposer davantage de rencontres Galago. [...] Je donne des rendez-vous à des gens quelque part et puis on joue tous ensemble. Et je trouve que ça donne un sens à tout mon travail solitaire. De pouvoir rencontrer des gens qui me regardent, et de pouvoir voir, de constater sur place qu'est-ce que ça donne par rapport à l'enseignement qu'ils ont pu recevoir. [...] Ça se fait un petit peu partout en France [...]. »

Un groupe privé Facebook Galagofan s'est créé à l'initiative d'abonnés de la chaîne YouTube. II compte 1899 membres. Parmi eux, certains publient régulièrement des vidéos de leurs prestations à domicile en se filmant avec leur téléphone portable le plus souvent, parfois avec un matériel d'enregistrement sonore un peu plus élaboré. Ce sont les progressions qui sont présentées la plupart du temps et l'esprit est toujours bienveillant (figures 2 et 3 ). 


\section{Raphaël ROTH et Laure-Hélène SWINNEN}

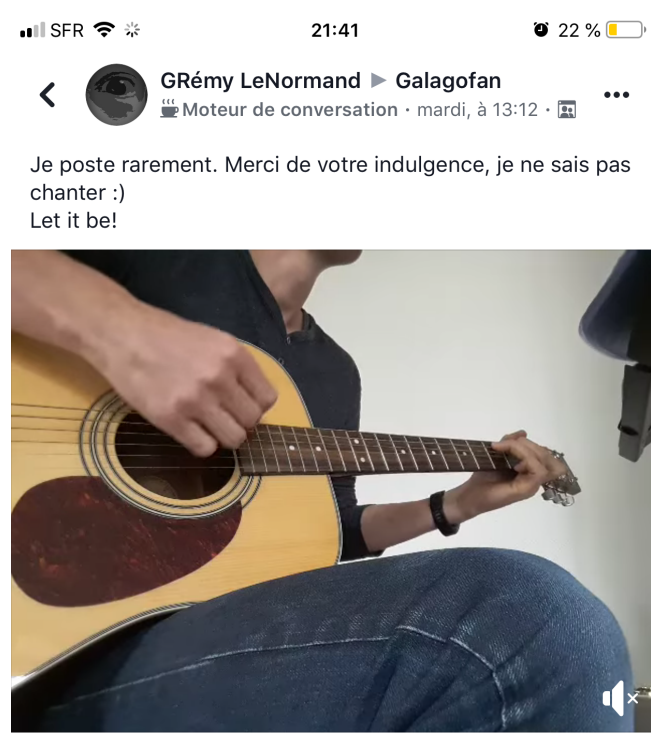

吸 J'aime

(1) 27

3. Michel Vellas

II n'y a pas d'indulgence à avoir, c'est très sympa, bravo..

(O) Votre commentaire..

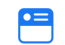

Figure 2. Capture d'écran de commentaires Facebook du groupe Galagofan

Ce groupe permet d'organiser les Galago rencontres mentionnées par Éric Legaud dans l'entretien. Elles fonctionnent comme une marque, une bannière qui porte l'« univers » et l'esprit de la chaîne YouTube MrGalagomusic. La bienveillance, le bon esprit et le plaisir de jouer et de progresser ensemble sont les maîtres-mots :

« II y en a une à Lille, à Lyon, à Marseille [...] au Havre. Je n'ai même pas besoin d'y aller, en fait, il suffit juste que quelqu'un dise qu'il connaît un lieu, les gens se rencontrent et je définis une espèce de process [...]. Le but du jeu, c'est qu'ils osent le faire comme moi j'aurais aimé oser le faire bien avant. Et ça marche. Les gens sont contents. » 
Enseigner la guitare sur YouTube. Le tutoriel gratuit et les contradictions de la " numérimorphose » de l'enseignement de l'instrument: le cas de la chaîne MrGalagomusic

«Et tout ça, sous l'égide Galago, sous le drapeau de Galago. Alors qu'en fait, ils pourraient faire juste une rencontre entre eux. Mais ce qui me flatte - parce que quand même j'ai toujours besoin de me rassurer - c'est d'avoir cette bannière Galago qui est comme référentielle [...]. »

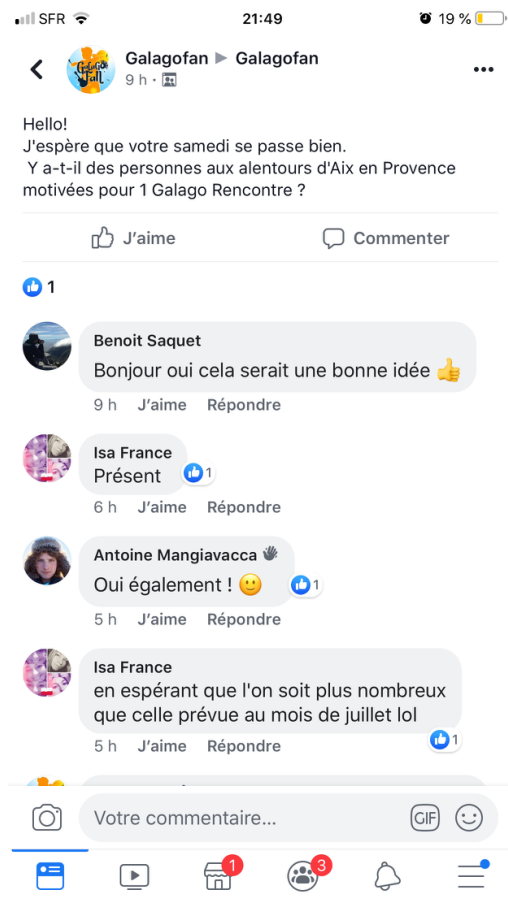

Figure 3. Capture d'écran de commentaires Facebook du groupe Galagofan

Ainsi, une communauté virtuelle se retrouve dans un espace public pour former une communauté physique. Pouvons-nous dès lors parler de "communauté d'apprentissage " où l'« apprendre ensemble » serait la motivation centrale ?

Pour qu'il y ait communauté, Katerine Bielaczyc et Allan Collins énumèrent huit conditions indispensables :

1. Un but commun de la communauté ; 2. Des activités d'apprentissage partagées, significatives et visibles ; 3 . Un rôle d'enseignant concepteur et animateur d'un espace d'apprentissage et des apprenants responsables de la conduite de leurs apprentissages individuels et collectifs et de leur propre évaluation; 4. Des changements de rôles 
des participants selon leurs connaissances et habiletés, chaque participant étant occasionnellement porteur ou demandeur de ressources; 5. Les ressources: les membres eux-mêmes et la collectivité ; 6 . Un langage commun co-élaboré au fur et à mesure des interactions entre les membres ; 7. Le consensus autour du savoir par approfondissement des idées; 8. La production par les apprenants de leurs propres objets et idées (cités dans Cristol, 2016, p. 155-163).

Dans ce cas précis, le groupe se réunit autour d'une logique de partage, d'une nécessité d'entraide et d'échanges de savoir grâce à de nouvelles méthodes et à un langage commun. Ce sont véritablement leurs objectifs et, dès lors, un sentiment d'appartenance à la communauté peut advenir. De nouvelles relations apparaissent entre les youtubeurs et leurs publics, possiblement par l'avènement de plusieurs formes de transmission: celles prises en charge par Éric Legaud, soit à travers un écran, soit en face à face, celles opérées entre pairs dans les commentaires ou sur les groupes Facebook, celles lors des Galago rencontres. II n'est pas rare d'observer spontanément des "changements de rôles" quand un participant devient, volontairement ou non, enseignant improvisé en fonction de ses connaissances, de ses compétences ou de ses expériences. Chacun met en commun ses efforts, son savoir et sa volonté ; tous prennent en compte les besoins, les avancées et les attentes de chacun. L'enseignement et l'apprentissage sont ainsi plus vivants, plus motivants et plus épanouissants.

Enseigner la guitare sur YouTube, c'est, pour Éric Legaud, prendre les qualités d'un bon professeur de guitare et les transmettre à un auditoire plus large, en conservant l'esprit du cours individuel.

\section{Cinquième contradiction : invariants et renouvellements de l'enseignement de l'instrument}

Dans un article publié pour l'encyclopédie Musiques, dirigée par Jean-Jacques Nattiez, la musicologue italienne Franca Ferrari (2004) décrit trois modèles de professeurs (qu'elle définit dans le cadre d'une formation musicale académique et 
Enseigner la guitare sur YouTube. Le tutoriel gratuit et les contradictions de la " numérimorphose » de l'enseignement de l'instrument: le cas de la chaîne MrGalagomusic

classique) «pour favoriser l'intégration entre éducation musicale et médias » (p. 906) : l'éclectique, l'ethnologue et le sympathisant.

Si cette catégorisation, comme toutes les tentatives de taxonomie, est forcément réductrice, elle offre tout de même, des orientations qui permettent de délimiter des qualités pédagogiques propres à l'enseignement de la guitare sur YouTube.

Ainsi, Éric Legaud semble correspondre au renouvellement d'une forme dynamique de ces trois figures. Comme l'éclectique,

il pense que la capacité de s'écarter des choix musicaux les plus fréquents en banals est un atout; il a pour référence non pas la musique, mais " les musiques » [...]. Pour l'éclectique, en fin de compte, toute chanson, tout jingle est prétexte à apprendre: un mécanisme, une astuce musicale (rythmique, mélodique, de timbre) que le jeune, en faisant le musicien, peut-être amené à découvrir par la simple imitation (Ferrari, 2004, p. 907).

Les apprenants "jouent comme " et " à la manière de ». L'imitation de l'enseignant est donc une forme d'intégration entre éducation musicale et média. II s'agit d'une imitation en abyme puisque l'enseignant pointe les " traits pertinents", les gestes et les façons de "sonner comme » le musicien en question (Ferrari, 2004).

L'ethnologue, quant à lui, s'interroge «sur l'opportunité de faire entrer en classe la musique des médias, et donc des jingles, les musiques de film et les chansons en tête du hitparade » (Ferrari, 2004, p. 908). Le professeur ethnologue adopte vis-à-vis de ses élèves une attitude compréhensive : il tient compte des rapports et des modes de relation qu'entretient son élève avec la musique qu'il aime écouter.

Le professeur sympathisant, enfin, fait systématiquement

de la classe un espace ouvert aux diverses conduites musicales, celles des jeunes comme celles de l'enseignant. Ainsi les musiques que les protagonistes eux-mêmes proposent - qui sont presque toujours acquises à travers les médias - fournissent l'occasion de 
construire en classe des espaces de sympathie, des projets de travail musical coopératif (p. 911).

Il a compris que, "plus il écoute ses élèves, plus il est en phase avec les motivations qui, surtout en dehors de l'école, les lient à la musique. Mieux, il réussit lui-même à produire des idées avec et autour des sons » (p. 911).

Dans sa formation musicale "classique " en conservatoire et en faculté de musicologie, Éric Legaud a probablement rencontré les trois types de professeurs décrits par Franca Ferrari. Les conservatoires et les écoles de musique en France aujourd'hui comptent de plus en plus de classes de musiques actuelles et les professeurs musiciens sont eux-mêmes en amitié avec un autre répertoire que le classique.

Nous pouvons, dès lors, envisager qu'Éric Legaud, en sa qualité de formateur, est impliqué dans un "dispositif hybride » (Bonfils, Dumas et Massou, 2014). En mobilisant différentes approches pédagogiques et en empruntant de nouvelles trajectoires, il propose à la fois des contenus personnalisés de formation à distance et de prise d'autonomie ainsi que des dispositifs participatifs (réseaux sociaux comme élément fédérateur des Galago rencontres). Le rapport à l'enseignement évolue notamment par une nouvelle " gestion des dimensions relationnelles et affectives entre formateurs et apprenants sous d'autres formes " (Bonfils, Dumas et Massou, 2014). En effet, Éric Legaud cultive avec empathie et vérité les rapports professeur/élève. II en parle en termes choisis :

«É : Vous voyez votre communauté comme des élèves [...]. Est-ce que c'est un choix de votre part de ne pas faire de distinguo entre les élèves de votre école et les spectateurs sur YouTube?

EL: Les personnes qui suivent les vidéos sont des personnes qui vont se mettre à un instrument [...] pour moi c'est juste normal d'appeler les gens qui me regardent des élèves. II n'y a pas de prétention. [...] J'enseigne quelque chose, un savoir qui me paraît important. Je ne mets pas de rapport de hiérarchie là-dedans [...]. Car les followers, je n'aime pas, les suiveurs non plus ${ }^{6}$. »

${ }^{6}$ Extrait d'un entretien entre Éric Legaud (EL) et un(e) étudiant(e) (É) en Master Culture et Communication à l'université d'Avignon. 
Enseigner la guitare sur YouTube. Le tutoriel gratuit et les contradictions de la " numérimorphose » de l'enseignement de l'instrument: le cas de la chaîne MrGalagomusic

« Et puis, j'aime donner des cours en vrai, parce que j'ai besoin de cet échange-là parce que j'apprends autant que ce que j'enseigne. Chaque élève est différent, il va t'apporter quelque chose de complémentaire en fait [...], je ne pourrai pas faire que du YouTube [...]. Même si je faisais des scores de dingue et que je gagnais ma vie super bien avec, je pense que j'aurais besoin des élèves [...]. »

On retrouve, dans les propos d'Éric Legaud, une posture qui associe les trois figures données par Franca Ferrari en pointant probablement un manque, qui est celui de la mise en confiance :

« Et j'ai voulu devenir, par mes vidéos YouTube, le prof que j'aurais voulu avoir. C'est-à-dire [...] un mec qui te prend la main et qui te dit: "ne t'inquiète pas, si j'y suis arrivé tout le monde peut y arriver, tu vas y arriver, tu es en train d'y arriver" [...]. Tu peux avoir un vrai pouvoir sur les émotions des gens si tu utilises un lexique de réalisation: tu vas y arriver [...]. Chaque élève est une aventure en fait. Chacun a ses craintes [...]. Tu crées ton atmosphère, tu crées l'univers qui permet à la personne de se sentir bien [...]. Et ça, c'est un truc que j'aime bien faire : faire en sorte que les gens soient bien, que ce soit en ma compagnie, que ce soit sur internet. »

II reste à se demander comment les "numérimorphoses ॥ de l'apprentissage de l'instrument sur YouTube modifient les postures et les attitudes, les relations que l'on connaît entre un apprentissage de l'instrument que nous qualifierons de " classique " (cours en présence du professeur, individuel, en conservatoire ou en école de musique) et l'apprentissage par l'intermédiaire d'une interface numérique (sur YouTube, par Skype ou tout autre logiciel de visioconférence, pack payant, etc.).

Le caractère ludique de la proposition d'Éric Legaud, sa sympathie, son écoute en matière de demandes de morceaux à travailler et son éclectisme sont évidents, mais en quoi relèvent-ils d'une qualité sur YouTube? 


\section{Conclusion : les vertus ludiques des tutos et leurs inspirations pour l'éducation musicale}

Les méthodes dites "actives " ont investi la pédagogie musicale depuis bien des années. Elles «se manifestent dans toute situation où l'élève est amené à faire de la musique bien avant d'en apprendre la théorie » (Dauphin, 2004, p. 833). Particulièrement adressées aux enfants, ces approches reposent sur

le rôle central du chant, des jeux rythmiques corporels et de la manipulation d'instruments simples. En fait il s'agit d'une organisation de la pédagogie musicale par la structuration et le développement des faits musicaux appartenant à l'univers traditionnel de l'enfance. Par là, la musique rejoint un autre principe fondamental des méthodes actives : faire de l'enfant le centre et le moteur même de sa propre évolution au lieu de le conditionner par la logique de l'adulte en devenir (p. 833).

Jouer avec la musique avant d'en jouer, c'est bien la posture d'apprentissage que proposent les tutoriels :

«RR: Est-ce que cela est né aussi d'une volonté pédagogique que de donner un autre moyen aux élèves d'apprendre et un regard un peu critique de l'école de musique et l'apprentissage du solfège ?

EL : Oui [...], je n'ai pas eu beaucoup de profs de guitare [...], j'ai appris dans les magazines [...], c'était toujours un peu compliqué. Donc, j'ai mis des années à apprendre des trucs que maintenant mes élèves apprennent en trois mois, parce qu'il y avait des formules que j'ai appris à découvrir soit tout seul, soit en regardant des tutos [...]. Et moi je me suis dit "pourquoi on ne me l'a jamais dit" alors que ça marche ! Donc, voilà, c'est des petits trucs que je pensais avoir en plus pour peut-être permettre aux gens d'arriver à jouer.

RR : Tu as tout à l'heure parlé du geste plutôt que de la théorie [...], pourquoi YouTube permet peut-être mieux ou différemment [...], est-ce que c'est un ingrédient de la transmission du geste? J'imagine que la présence en face à face d'un prof et son élève est la meilleure configuration pour transmettre un geste. 
Enseigner la guitare sur YouTube. Le tutoriel gratuit et les contradictions de la " numérimorphose » de l'enseignement de l'instrument: le cas de la chaîne MrGalagomusic

EL : Bien sûr. De toute façon la musique, c'est quand même un art de tradition orale, c'est-à-dire qu'on a pas besoin de savoir lire une partition pour arriver à jouer une chose. [...] On a Django Reinhardt, Jimi Hendrix [...], il y en a plein des exemples de mecs qui ne savent pas du tout lire une partoche, qui se débrouillent quand même et qui ont appris en regardant le voisin ou le copain [...], en guitare notamment [...], c'est hyper important de repérer ce qui se passe. Parce que tout intellectualiser, ce n'est pas forcément une bonne idée [...], il est très important d'arriver à choper les gestes [...]. Et c'est vrai que YouTube permet ça, du coup, parce qu'en faisant un gros plan, on a une meilleure visibilité [...]. On peut en plus arrêter la vidéo, recommencer autant de fois qu'on veut. Donc, c'est vrai que la pratique du geste est quelque chose qui m'est cher et qui me semble être un des fondamentaux pour le cas des enseignements sur YouTube [...]. Pour le mien, en tout cas !

RR: Avoir un prof en permanence chez soi sur son ordinateur, c'est mieux que d'avoir un prof...?

EL : Je pense que c'est bien d'avoir les deux [...]. Le prof sur YouTube ne remplacera jamais la personne en face à qui tu peux poser des questions, avec qui tu peux discuter et créer aussi une complicité [...]. Les vidéos sont un petit truc en plus. C'est vraiment si on n'a pas de sous, si on a pas le temps et qu'on n'arrive pas à en sortir. »

Les travaux de David W. Johnson et de Roger T. Johnson (1974) rejoignent ce propos. Ils définissent trois manières d'apprendre: l'individuelle, la collaborative et la compétitive, sans pour autant les mettre en opposition. Ils précisent que les trois structures sont utiles à connaître et à manier en fonction des objectifs pédagogiques recherchés. Ceci nous amène à nous demander comme Éric Legaud envisage la transmission de son message face à des audiences hétéroclites n'ayant pas les mêmes connaissances, pas la même histoire, pas le même niveau de pratique, pas les mêmes capacités d'écoute, pas les mêmes aspirations et surtout pas les mêmes goûts :

« II y a des gens qui n'ont jamais touché une guitare et qui arrivent maintenant à jouer et chanter en même temps, que ce soit des ados, des adultes, des seniors d'ailleurs. II 
y en a une qui est géniale, elle s'appelle Uvie, elle doit avoir 60, 65 ans je crois. Elle a commencé il y a deux ans [...] elle a suivi mon tuto d'un morceau d'Eminem qui s'appelle Loose yourself. "

«É : Vous êtes capable de savoir s'il y a des jeunes qui regardent vos vidéos YouTube?

EL : Oui, il y en a plein. Ce qui est rigolo, c'est que des fois c'est les jeunes qui commencent et du coup les parents disent "ouais tu joues bien!". Des fois, c'est les parents qui s'y mettent. Du coup, c'est assez familial, vraiment family/friendly. "

Il y a donc bien une vertu ludique des tutoriels sur YouTube qui joue grandement, outre l'aspect gratuit de la proposition, dans la soif d'apprendre et de progresser à l'instrument de manière collective.

Le joujou est la première initiation de l'enfant à l'art, ou plutôt c'en est pour lui la première réalisation, et, l'âge mûr venu, les réalisations perfectionnées ne donneront pas à son esprit les mêmes chaleurs, ni les mêmes enthousiasmes, ni la même croyance (Baudelaire, 1863, p. 143).

Comme les jouets de Baudelaire, le tutoriel sur YouTube agit sur les élèves. II agit d'autant mieux sur eux qu'ils le comprennent. Pour le comprendre, comme le jouet, ils ont besoin de le déconstruire, de le manipuler, de le maltraiter pour en saisir l'« âme ». L'éducation musicale passe par cette nécessité de casser le joujou, de le déconstruire pour comprendre comment il fonctionne. Cela participe de la construction de l'esprit critique et révèle ce désir d'art et de culture afin d'alimenter les «formes les plus délicieuses du beau », pour emprunter ici la formule de Baudelaire.

Certes, il ne faut ni oublier ni nier en reconnaissant la vertu des industries culturelles et des plateformes, la primauté de leur objectif : générer de la valeur par la vente des produits culturels au plus grand nombre de consommateurs. II ne faut pas non plus, chemin faisant, négliger l'importance des signes et des messages que les médias de masse véhiculent et leurs effets sur les publics les plus jeunes notamment, car rien n'est vertueux pour qui n'est pas éduqué et sur le chemin de 
Enseigner la guitare sur YouTube. Le tutoriel gratuit et les contradictions de la « numérimorphose » de l'enseignement de l'instrument : le cas de la chaîne MrGalagomusic

l'émancipation. Une fois décrite, cette réalité ne doit cependant pas effacer la valeur symbolique du cinéma, de la musique, de la bande dessinée, des jeux vidéo ou de YouTube dans leur complexité, y compris artistique ou pédagogique. Le divertissement qu'ils procurent ne peut reléguer au second plan la valeur des productions issues des plateformes, car elles sont un maillon de la chaîne qui relie l'œuvre à ses publics.

\section{Références}

Adorno, T. W. (1964). L'industrie culturelle. Communications, (3), 12-18. https://doi.org/10.3406/comm.1964.993

Adorno, T. W. (2010). Current of Music. Paris, France : Maison des sciences de l'homme.

Baudelaire, C. (1853, 17 avril). Morale du joujou. Le Monde littéraire (p. 143).

Benjamin, W. (2014). Écrits radiophoniques. Paris, France: Éditions Allia.

Bonfils, P., Dumas, P. et Massou, L. (dir.) (2014). Numérique \& Éducation. Dispositifs, jeux, enjeux, hors-jeux. Questions de communications, (34).

Cristol, D. (2016). Peut-on créer des communautés d'apprentissage ?. Éducation permanente, (207), 155-163.

Dauphin, C. (2004). Les grandes méthodes pédagogiques du $X X^{\mathrm{e}}$ siècle. Dans J.-J. Nattiez (dir.), Musiques. Une encyclopédie pour le XXle siècle. 2. Les savoirs musicaux (p. 833-853). Arles, France : Actes Sud.

Ethis, E. (2005). Sociologie du cinéma et de ses publics. Paris, France : Armand Colin.

Ferrari, F. (2004). Éducation musicale et médias. Dans J.-J. Nattiez (dir.), Musiques. Une encyclopédie pour le XXI siècle. 2. Les savoirs musicaux (p. 899-912). Arles, France : Actes Sud.

Flichy, P. (2010). Le sacre de l'amateur. Sociologie des passions ordinaires à l'ère numérique. Paris, France : Seuil. 
Jankélévitch, V. (2009). La Musique et l'Ineffable. Paris, Fance : Seuil.

Jenkins, H. (2006). Fans, Bloggers, and Gamers: Exploring Participatory Culture. New York, NY: New York University Press.

Johnson, D. W. et Johnson, R. T. (1974). Instructional Goal Structure: Cooperative, Competitive or Individualistic. Review of Educational Research, 44(2), 213-240.

Jost, F. (1997). La promesse des genres. Réseaux, 15(81), 1131.

Le Guern, P. (dir.) (2016). Où va la musique ? Numérimorphose et nouvelles expériences d'écoute. Paris, France: Presses des Mines.

Leveratto, J. -M., Pourquier-Jacquin, S., Roth, R. (2015). Voir et se voir: le rôle des écrans dans les festivals de musique amplifiée. Culture \& Musées, (24), 23-41.

Malinas, D. (2008). Portraits des festivaliers d'Avignon: Transmettre une fois ? Pour toujours?. Grenoble, France: Presses universitaires de Grenoble.

Miège, B. (2017). Les industries culturelles et créatives face à l'ordre de l'information et de la communication. Grenoble, France: Presses de l'Université de Grenoble.

Paveau, M.-A. (2017). L'Analyse du discours numérique. Dictionnaire des formes et des pratiques. Paris, France: Hermann.

Passeron, J.-C. (2006). Le raisonnement sociologique. Paris, France : Albin Michel.

Passeron, J. -C. et Pedler, E. (2009). Du musée aux tableaux. Idées économiques et sociales, (155), 12-18. doi: 10.3917/idee.155.0012

Passeron, J.-C. et Revel, J. (dir.). (2005). Penser par cas. Paris, France: Éditions de l'École des hautes études en sciences sociales. 
Enseigner la guitare sur YouTube. Le tutoriel gratuit et les contradictions de la « numérimorphose » de l'enseignement de l'instrument : le cas de la chaîne MrGalagomusic

Roth, R. et Dufour, R. (2020). Apprendre la guitare sur YouTube. Partages et échanges, contributions et participations autour des tutoriels d'apprentissage de l'instrument. Interfaces numériques, 9(2). Repéré à http://dx.doi.org/10.25965/interfaces-numeriques.4288

Sterne, J. (2003). The Audible Past. Cultural origins of sound reproduction. Durham/ London : Duke University Press.

\section{Remerciements}

Les auteurs remercient Emmanuel Ethis et Damien Malinas pour leurs précieuses relectures de cet article et leurs contributions éclairées. Merci également à Richard Dufour, maître de conférences en informatique du laboratoire informatique d'Avignon pour la collecte et la structuration des données et Christlord Forrest, étudiante en master Culture et Communication à l'université d'Avignon pour la retranscription des entretiens. Merci également à Éric Legaud, et aux abonnés de sa chaîne YouTube, pour sa disponibilité lors des entretiens, et aux étudiants du master Culture et Communication de l'université d'Avignon pour leurs contributions à la réalisation d'un des entretiens. 